\title{
SATURATION MEASUREMENTS OF A VISIBLE SASE FEL*
}

\author{
A. Tremaine ${ }^{1}$, P. Frigola, A. Murokh, C. Pellegrini, S. Reiche, J. Rosenzweig \\ University of California, Los Angeles, CA, 90095
}
M. Babzien, I. Ben-Zvi, E. Johnson, R. Malone, G. Rakowsky, J. Skaritka, X.J. Wang, V. Yakimenko Brookhaven National Laboratory, Upton, NY, 11973
L. Bertolini, J. Hill, G. Le Sage, M. Libkind, A. Toor, K.Van Bibber, Livermore National Laboratory, Livermore, CA., 94551

\author{
R. Carr, M. Cornacchia, L. Klaisner, H.-D. Nuhn, R. Ruland \\ Stanford Linear Accelerator Center, Stanford, CA, 94309
}

\begin{abstract}
VISA (Visible to Infrared SASE Amplifier) is an FEL designed to obtain high gain at a radiation wavelength of $800 \mathrm{~nm}$. Large gain is achieved by driving the FEL with the $71 \mathrm{MeV}$, high brightness beam of the Accelerator Test Facility (ATF) and using a novel, strong focusing, $4 \mathrm{~m}$ long undulator with a gap of $6 \mathrm{~mm}$ and a period of $1.8 \mathrm{~cm}$. We report measurements of exponential gain, saturation, and spectra of the FEL radiation intensity.
\end{abstract}

\section{INTRODUCTION}

VISA, an R\&D experiment for the Linac Coherent Light Source (LCLS), is an $800 \mathrm{~nm}$ SASE FEL that has been designed to demonstrate high gain and saturation using an electron beam with a brightnesss similar to that needed for a $1 \AA$ X-ray FEL $[1,2]$. We present in this paper a description of the experimental setup, which includes the electron beam source, transport beamlines and undulator magnet. Finally, measurements demonstrating saturation with only $3.6 \mathrm{~m}$ of undulator and a gain length of $18.7 \mathrm{~cm}$ are presented.

\section{EXPERIMENTAL SETUP}

Table 1 shows the VISA FEL design parameters

Table 1. VISA FEL Design Parameters

\begin{tabular}{|l|l|}
\hline Wavelength (Fundamental) $\lambda$ & $840 \mathrm{~nm}$ \\
\hline E-beam Energy, E & $71 \mathrm{MeV}$ \\
\hline Peak Current, I & $200 \mathrm{~A}$ \\
\hline Emittance, $\varepsilon$ & $2 \mathrm{~mm}-\mathrm{mrad}$ \\
\hline Power Gain Length, L & $18.7 \mathrm{~cm}$ \\
\hline Spot size, $\sigma$ & $60 \mu \mathrm{m}$ \\
\hline
\end{tabular}

important to this paper. VISA uses the high brightness photoinjector system of the ATF [3] and a novel permanent magnet undulator [4]. A 1.6-cell S-band gun, emittance compensating solenoid and two SLAC type linac sections deliver a high brightness beam to the VISA undulator.

Between the linac section and the VISA undulator, the beam propagates through a series of transport lines. The ATF matching section immediately follows the linac line. Nine quadrupole magnets are used for beam diagnostics in addition to matching the beam to VISA and other beamline experiments. After the matching section, the beam propagates through the transport line. The optics in this line include two 20 degree bending magnets and eight quadrupoles in between the bends. These components form an achromatic transport line and we discuss below the possibility of strong compression and thus, an increase in beam current. The VISA matching just before the undulator has three quadrupoles that provide the final focusing and matching into the undulator.

The VISA undulator is a Hallbach array, and strong focusing is introduced by placing dipole magnets on either side of the electron beam propagation axis [5]. Four $1-\mathrm{m}$ undulator sections with a $1.8 \mathrm{~cm}$ period and $6 \mathrm{~mm}$ gap are butted up against one another and situated in a vacuum vessel during operation. Simulations [6] show significant reduction in FEL performance if these sections are not aligned [7] to within $50 \mu \mathrm{m}$. In addition, access ports with only a $3.7 \mathrm{~mm}$ aperture have made implementation of the intra-undulator diagnostics challenging [8]. It should be noted that the undulator must be aligned at atmospheric pressure, after which the vacuum tank is closed up and pumped down.

\footnotetext{
*Work supported by the U.S. Department of Energy (DOE) contract DEAC03-76SF00515.

${ }^{1}$ tremaine@physics.ucla.edu
} 
A description of the diagnostics along the length of the undulator are elsewhere [8] but are briefly reviewed here because of their importance to this experiment. VISA has pop-in diagnostic ports every $50 \mathrm{~cm}$, giving a total of 8 stations along the $4 \mathrm{~m}$ undulator. The last port is $25 \mathrm{~cm}$ before the end of the undulator. Each port serves two purposes: a beam position monitor and an FEL radiation monitor. We found that SASE signals above spontaneous could be detected from the last 6 pop-in monitors when the system was in saturation.

Improvements were made to the undulator after the initial commissioning of the VISA FEL. One was the installation of an optical monitoring system which allows the position of the undulator to be monitored to within 10 $\mu \mathrm{m}$ [9]. During the next pump down, the undulator was observed to move by up to $500 \mu \mathrm{m}$ in the horizontal direction. This amount of movement was enough to misalign the undulator to a point where only a gain of 100 was measured [10]. After attempts to straighten the undulator while it was under vacuum had not resulted in increased gain, the undulator supports were strengthened to reduce the movement during pump down. No motion was observed during the next pump down, and a gain of $10^{3}$ was immediately measured afterwards.

Next, the matching beam was studied in order to optimize the beam optics. Since the VISA undulator does not have a half period, but a full period at the entrance to the undulator, the correct match is found to be $\beta_{x, y}=.230, \alpha_{x}=-1.75$, and $\alpha_{y}=1.75$. Once these values were achieved, gain of $10^{6}$ was measured and shortly thereafter, saturation obtained.

\section{MEASUREMENTS}

The most direct method to measure saturation is to study the SASE energy vs. distance along the undulator. If in saturation, the SASE energy towards the end of the undulator will deviate from the exponential gain measured at the preceding positions. Figure 1 shows a measurement of the SASE energy vs. distance at $170 \mathrm{pC}$.

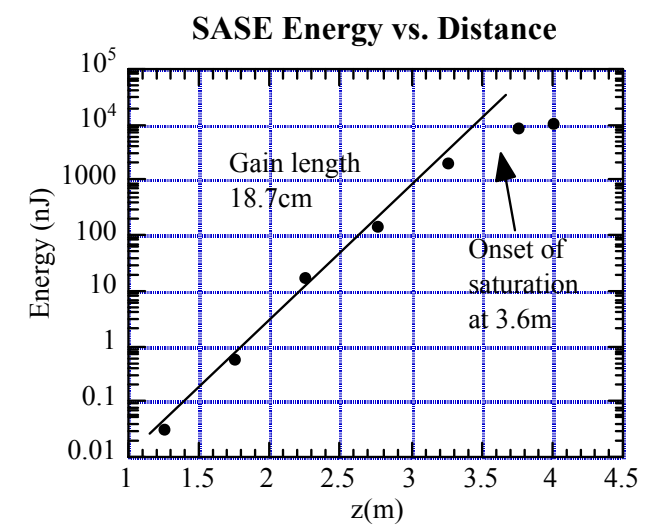

Figure 1. SASE Energy vs. Distance. Onset of saturation occurs at about $3.6 \mathrm{~m}$ and a power gain length of $18.7 \mathrm{~cm}$ is measured.
It can be seen from Fig. 1 that saturation begins at $3.6 \mathrm{~m}$. Excluding the last two points in Fig.1, a power gain length of $18.7 \mathrm{~cm}$ is calculated from the line fit shown in the figure. It should be noted that a gain length of $8 \mathrm{~cm}$ was measured in another SASE experiment at a wavelength of $13 \mu \mathrm{m}$ [11], but to date the gain length reported here is the shortest measured for a SASE FEL in the visible spectral region.

An estimate for the total gain of the system can be made. Defining gain as

$$
G=\frac{E}{E_{0}}
$$

where $E$ is the total energy measured at the undulator exit and $E_{0}$ is the spontaneous energy inside the coherency cone and bandwidth for the first gain length, we calculate a gain, $G=2 * 10^{7}$. $E_{0}$ can be determined from the data given in Fig.1. A continuation of the line to the first gain length (about $18.5 \mathrm{~cm}$ from the entrance of the undulator) represents the seed for the SASE process and thus, the spontaneous emission in the coherency cone and bandwidth. This energy matches that which can be theoretically calculated, also resulting in the gain stated above.

Next, the spectrum was measured and is shown in Fig. 2.

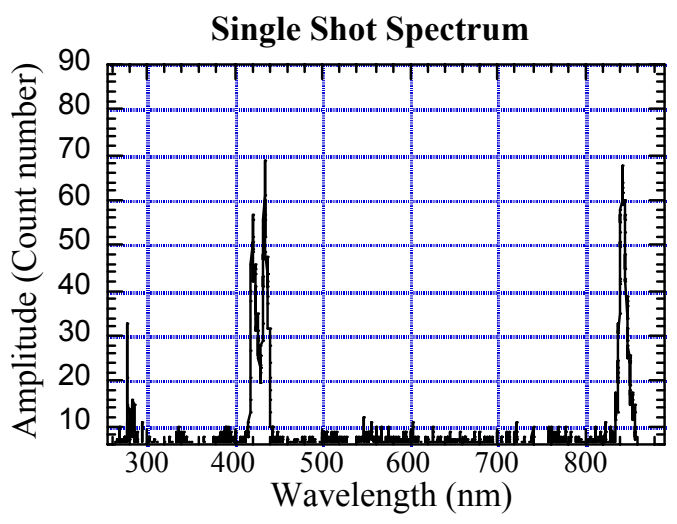

Figure 2. High power single-shot SASE spectrum captured at gain $>10^{6}$. Shown are the Fundamental, $2^{\text {nd }}$ and $3^{\text {rd }}$ harmonic modes at $845 \mathrm{~nm}, 422 \mathrm{~nm}$, and $280 \mathrm{~nm}$, respectively.

It should be mentioned that the spectrometer highly attenuates the IR, peaks in sensitivity at $300 \mathrm{~nm}$, and has a resolution of $1.5 \mathrm{~nm}$. Using the FEL equation

$$
\lambda=\frac{\lambda_{u}}{2 \gamma^{2}}\left(1+\frac{K^{2}}{2}\right)
$$


where $K=1.29$ is the undulator parameter, $\gamma=71$ is the energy and $\lambda_{u}=1.8 \mathrm{~cm}$ is the undulator period, we expect a fundamental wavelength of $845 \mathrm{~nm}$. The measured spectrum is nearly centered at $840 \mathrm{~nm}$ as seen in Fig. 2. Only one peak is seen for the fundamental at high gain. This is an indication that the electron beam length might be of about the same magnitude as $2 \pi L_{C}$ where $L_{C}$ is the cooperation length. A further discussion on the pulse length is given below. Strong coupling to the $2^{\text {nd }}$ and $3^{\text {rd }}$ harmonics shown in Fig. 2 is additional evidence that the system is in high gain. A future publication will detail the harmonic characteristics of this SASE FEL system.

The FWHM of the fundamental spectrum above is 10 $\mathrm{nm}$, compared with an expected value $\lambda / \mathrm{Nw} \sim 4 \mathrm{~nm}$, where $N_{w}=220$ is the number of undulator periods. The discrepancy comes from the fact that in order to obtain high gain, an energy chirp is put on the beam in the linac line. Measurements show an energy spread of $\sim .3 \%$; a result leading to spectral broadening.

A strong dependence of gain on the linac phase is observed for good system performance. This dependence and the spectral width give us a strong indication that chirping in the linac and compression in the dispersion region give a current larger than the $75 \mathrm{~A}$ measured at the linac. Because the beam parameters have such importance in this experiment, bunch length at the undulator became an important issue.

Preliminary bunch length measurements using coherent transition radiation (CTR) after the VISA undulator indicate that the electron bunch may be shorter than what was measured in the linac line. PARMELA and ELEGANT have bee used to simulate the ATF photoinjector and beamline. Results show the possibility of beam compression in the dispersion section and a peak current greater at the undulator than that measured after the linac section. Additionally, these simulations show preservation of the vertical emittance, and a modest increase in the horizontal emittance. More analysis is currently being pursued on the pulse length data; however, the beam quality delivered to the VISA undulator in either scenario would be good enough to drive a SASE X-ray facility.

Because the beam properties are very important to the VISA experiment, careful examination must be done on the recent beam measurements. Detailed analysis is currently being completed and a comparison with theory and simulation will be presented in a future publication.

\section{CONCLUSION}

We have characterized the VISA FEL in saturation. VISA has demonstrated the shortest gain length in addition to the shortest saturation length measured for a SASE FEL in the visible spectral region. Spectral measurements show strong coupling to the $2^{\text {nd }}$ and $3^{\text {rd }}$ harmonic radiation and are further indication that high gain has been obtained. Furthermore, to attain this high level of performance, the beam delivered to the VISA undulator does have the brightness necessary to drive very short wavelength FELs.

\section{REFERENCES}

[1] M. Cornacchia, Performance and Design Concepts of a Free Electron Laser Operating in the X-ray Region, March 1997, SLAC-PUB-7433.

[2] J.B. Murphy and C. Pellegrini, 1985, Nucl. Instrum. \& Methods Phys. Res. A 237, 108.

[3] X.J.Wang et al., Proceedings 1999 Particle Accelerator Conference, New York, NY.

[4] M. Libkind et al., 1999 Particle Accelerator Conference, New York, NY.

[5] A.A. Varvolomeev and A.H. Hairetdinov, Nucl. Inst. \& Methods A341, 1994.

[6] P. Emma et al., Proceedings of the 20th International

FEL Conference (FEL98), Williamsburg, Va., USA, 1998, SLAC-PB-7913.

[7] R. Ruland et al., Proceedings 1999 Particle Accelerator Conference, New York, New York, 1999

[8] A. Murokh et al. at these proceedings.

[9] Tremaine et al., BNL Informal Report 68170.

[10] Tremaine et al., $22^{\text {nd }}$ International FEL Conference

(FEL 2000), Durham, N.C., USA, 2000.

[11] M.Hogan et al., Phys. Rev. Lett., 80, 298 (1998). 